\title{
Filtre optique accordable par interaction acousto-optique colinéaire à ondes de surface
}

\author{
C. ZUCCHI, E. BIGLER* et A. ZAREMBOWITCH
}

Département de Recherches Physiques, Université Pierre et Marie Curie, T. 22, 4 place Jussieu, 75252 Paris cedex 05, France

* Laboratoire de Physique et Métrologie des Oscillateurs, Université de Franche-Comté, 32 av. de l'Observatoire, 25000 Besançon, France

\begin{abstract}
Acousto-optical mode convecters are the basic elements for a variety of integrated optical circuits. It has been demonstrated that efficient acousto-optical mode converters with a large tuning range and low drive power can be realized in a combined structure of acoustical and optical waveguides. We use an acoustical directional coupler to obtain a weighted coupling resulting in a theorical filter response with large sidelobe suppression. Nearly $100 \%$ TE/TM polarization conversion efficiency was achieved using only $3 \mathrm{~mW}$ of applied RF power in a $1.52 \mu \mathrm{m}$ filter of $2 \mathrm{~nm}$ optical bandwidth on a cristal of $\mathrm{LiNbO}_{3}$. Yet, the absolute sidelobe levels were significantly higher than expected. This is presumably due to nonuniformity in the effective birefringence of the optical waveguides.
\end{abstract}

Introduction :

Les dispositifs permettant des conversions de mode TE/TM sélectives en longueur d'onde, sont appelés à remplir des fonctions importantes en optique intégrée : filtrage, modulation, commutation, ou multiplexage.

Dans le cas d'effets acousto-optiques, une interaction dont les vecteurs d'onde sont colinéaires présente de multiples avantages : 1/ une grande accordabilité (10 fois plus importante qu'un dispositif électro-optigue fonctionnant sur le même principe), 2/ une grande longueur d'interaction permettant d'obtenir un rendement de conversion TE/TM de $100 \%$ pour de très faibles puissances de commande (< $10 \mathrm{~mW}$ électrique), 3/ la possibilité de géométries complexes favorisant l'apodisation des lobes secondaires du filtre en utilisant des transducteurs focalisés, des guides acoustiques, ou encore des guides acoustiques couplés [1], 4/ une intégration avec d'autres composants optiques sur niobate de lithium (polariseurs, coupleurs optiques, modulateurs électro-optiques).

\section{Principe de fonctionnement :}

Une interaction acousto-optique est réalisée à la surface d'un cristal de niobate de lithium $\mathrm{LiNbO}_{3}$ (cristal uniaxe et piézo-électrique) entre une onde optique guidée monomode polarisée, et une onde acoustique de Rayleigh (onde de surface) de vecteur d'onde colinéaire au vecteur d'onde optique. Les conditions d'interaction colinéaire sont sélectives, elles sont liées à la conservation des vecteurs d'ondes $\left(\vec{k}_{T E}=\vec{k}_{T M} \pm \vec{K}_{a c}\right)$ et sont donc obtenues si $F_{a}=V_{a} \frac{n_{e}-n_{0}}{\hat{\lambda}}$ où $F_{a}$ et $V_{a}$ sont respectivement la fréquence et la vitesse de l'onde acoustique de surface, $n_{e}$ et $n_{0}$ les indices extraordinaire et ordinaire du cristal, et $\lambda$ la longueur d'onde optique. A la suite de cette interaction, si les coefficients élasto-optiques sont favorables, l'onde lumineuse TE (transverse électrique) est convertie partiellement en une onde TM (transverse magnétique) qui peut être transmise seule par le dispositif intégré si un polariseur élimine l'onde TE résiduelle. L'onde ainsi transmise est une onde filtrée autour de la longueur d'onde optique d'accord déduite de la relation précédente. Il y a accordabilité de la longueur d'onde optique filtrée par modification de la fréquence acoustique : $\frac{\Delta F_{3}}{F_{a}}=\frac{\Delta \lambda}{\lambda}$. On obtient typiquement une accordabilité de $8.5 \mathrm{~nm} / \mathrm{MHz}$ autour de $\lambda=1.52 \mu \mathrm{m}$.

A partir des expressions complexes des champs électromagnétiques de l'onde optique, ainsi que de l'expression de la déformation acoustique, il est possible d'exprimer simplement [2] le rendement de 
l'interaction $\eta$ qui est le rapport entre la puissance optique diffractee lors de l'interaction, et la puissance optique d'entrée. Après une longueur d'interaction $L$, on obtient

$$
\eta=\frac{P_{\mathrm{TM}}(\mathrm{L})}{\mathrm{P}_{\mathrm{TE}}(0)}=\frac{\omega_{\circ}}{\omega_{e}} \Gamma^{2} L^{2} \sin c^{2}\left(\sqrt{\Gamma^{2} L^{2}+\left(\frac{\Delta K}{2}\right)^{2} L^{2}}\right)
$$

où $\Delta K=k_{0}-k_{e}-K_{a}$ représente l'accord de phase, et $\Gamma^{2}=\frac{\pi^{2}}{2 \lambda^{2}} M \frac{P_{a}}{A}$ le coefficient de couplage de l'interaction. $M=\frac{n_{o}^{3} n_{c}^{3} P^{2}}{\rho V_{a}^{3}}$ est le facteur de mérite de l'interaction avec $P$ coefficient élasto-optique, $\rho$ densité de volume du cristal, $\mathrm{P}_{\mathrm{a}}$ puissance acoustique et $\mathrm{A}$ surface d'émission de l'onde acoustique.

D'après l'équation $\{1\}$, le maximum de transmission du filtre est atteint pour un accord de phase parfait, c'est à dire $\Delta K=0$, et l'on obtient un rendement de $100 \%$ pour la condition $\Gamma L=\frac{\pi}{2}$ (notons que le rapport $\omega_{\mathrm{o}} / \omega_{\mathrm{e}}$ est proche de 1 et peut être négligé).

\section{Approche expérimentale :}

De tels filtres acousto-optiques ont été réalisés à la surface du niobate de lithium tout d'abord en coupe $Y$ et axe de propagation suivant $X$, le coefficient élasto-optique le plus important $p_{41}=0.155$ de $\mathrm{LiNbO}_{3}$ intervenant dans l'interaction via la contrainte longitudinale $\mathrm{S}_{11}$ de l'onde de surface. Mais depuis 1988, il est apparu plus intéressant d'exploiter la contrainte transversale $S_{12}$ (plus importante que $S_{11}$, et toujours liée à $\mathrm{p}_{41}$ ) de l'onde de Rayleigh se propageant suivant $\mathrm{Y}$ à la surface du niobate en coupe $X$. De plus, cette orientation permet d'obtenir un meilleur couplage électroacoustique $\mathrm{K}_{\mathrm{r}}^{2}=1.75 \mathrm{e}^{-2}$ que la précédente $\left(0.78 \mathrm{e}^{-2}\right)$ pour la génération de l'onde de Rayleigh par les peignes interdigités. Par contre, une déviation de $5^{\circ}$ apparaît sur le flux d'énergie de l'onde de surface dans le cas d'une coupe $\mathrm{X}-\mathrm{Y}$, et oblige à une compensation par rotation des peignes interdigités.

Une largeur à mi-hauteur de $1.3 \mathrm{~nm}$, avec un rendement de l'ordre de $97 \%$, et une puissance électrique de commande de $100 \mathrm{~mW}$, a été atteinte [3] en choisissant cette orientation, mais le problème du niveau important des lobes secondaires à $-8 \mathrm{~dB}$ est apparu (le niveau théorique prévoyant $-9.7 \mathrm{~dB}$ ). Pour remédier à ce problème lié à des échauffements locaux parasites sous les peignes interdigités ou sous les absorbants [4], la réalisation de guides acoustiques permettant de réduire de façon drastique la puissance de commande électrique s'est révélée nécéssaire. Une nouvelle réalisation [5] avec des guides acoustiques de largeur $100 \mu \mathrm{m}$, a permis de réduire jusqu'à $8 \mathrm{~mW}$ la puissance de commande, mais n'a pas réduit pour autant le niveau des lobes secondaires $(-7.5 \mathrm{~dB}){ }^{1}$.

\section{Guides acoustiques couplés :}

Les solutions que nous avons envisagées, font appel à la réalisation de guides acoustiques couplés [6], qui présentent de multiples avantages par rapport aux solutions précédentes. En rapprochant deux guides acoustiques suffisamment près l'un de l'autre, nous pouvons en émettant une onde acoustique dans un des guides, la faire se propager entièrement (au bout d'une longueur $\mathrm{L}_{c}$, dite longueur de couplage) dans le guide adjacent par couplage de modes symétriques et antisymétriques. En plaçant le guide optique au centre d'un des guides acoustiques, et en émettant l'onde de surface dans l'autre guide, l'onde optique lors de sa propagation dans le cristal ne sera pas perturbé par les échauffements dus aux transducteurs et aux absorbants, et de plus l'interaction acousto-optique sera réalisée sur une longueur de $2 . \mathrm{L}_{\mathrm{c}}$ par une onde acoustique dont l'amplitude varie en $\sin ^{2}$ (voir figure $n^{\circ} 1$ ). La forme en $\sin ^{2}$ de la déformation acoustique permet d'apodiser de façon importante le spectre du filtre acousto-optique (le niveau du premier lobe secondaire passant à -17.4 dB). Par contre, cette apodisation se fait au détriment de la largeur à mi-hauteur du filtre qui augmente d'un facteur 1.4, et de la puissance de commande qui elle aussi augmente d'un facteur $(\pi / 2)^{2}$ (voir figure $n^{\circ} 2$ ).

${ }^{1}$ Bien qu'il y est diminution de la puissance acoustique, il y a concentration géographique de l'échauffement (sur une largeur de $100 \mu \mathrm{m})$ au dessus du guide optique. 


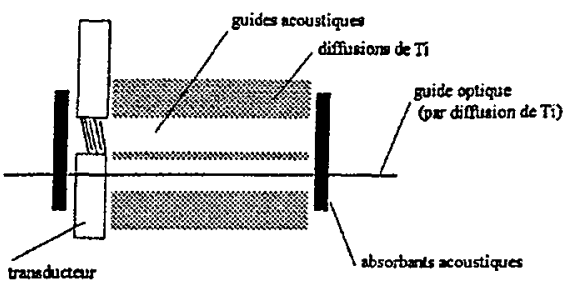

Figure $n^{\circ} 1:$ Schéma de principe du composant avec un guide acoustique couplé.

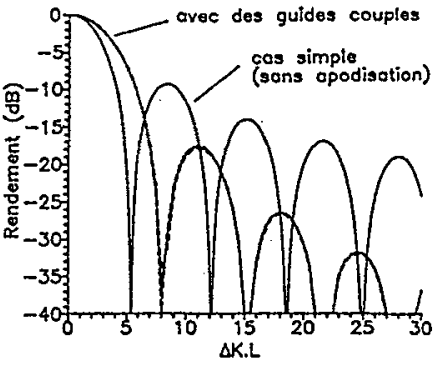

Figure $n^{\circ} 2$ : Spectre théorique du filtre acousto-optique ${ }^{2}$.

Réalisations et résultats :

Nos dispositifs ont été réalisés sur des cristaux de $\mathrm{LiNbO}_{3}$ en coupe $\mathrm{X}$. Une première étape consistait à réaliser les guides acoustiques par diffusion d'une couche de titane d'épaisseur $1600 \AA$ pendant $35 \mathrm{~h} \mathrm{a} 1050^{\circ} \mathrm{C}$ de part et d'autre de ceux-ci. La diminution de la vitesse de l'onde de surface de l'ordre de $1 \%$ induite par cette diffusion, permet ainsi de former les guides acoustiques dans les parties non-diffusées. Trois types de guides acoustiques couplés différents ont été réalisés et testés (chacun d'eux est formé de deux guides d'égale largeur $W$ séparés par un gap de largeur $G$, en jouant sur $W$ et $G$ nous obtenons des longueurs de couplage différentes),

\begin{tabular}{|c|c|c|c|}
\hline & $1^{\text {er }}$ cas & $2^{\text {eme }}$ cas & $3^{\text {eme }}$ cas \\
\hline$W(n m)$ & 70 & 100 & 100 \\
\hline$G(n m)$ & 20 & 20 & 40 \\
\hline$L_{c}(\mathrm{~mm})$ & 6.75 & 9.5 & 13.5 \\
\hline
\end{tabular}

La deuxième étape consistait a réaliser un guide optique au centre d'un des guides acoustiques. La même technique de diffusion de titane permet de réaliser des guides monomodes gaussiens à 1.52 $\mu \mathrm{m}$ à partir d'une ligne d'épaisseur $800 \AA$ et de largeur $8 \mu \mathrm{m}$ diffusée pendant $11 \mathrm{~h} \mathrm{à} 1030^{\circ} \mathrm{C}$ (induisant une variation des indices optiques du niobate de l'ordre de $\left.2 \mathrm{e}^{-3}\right)^{3}$.

Après un polissage des faces d'entrée et sortie des guides optiques, les transducteurs formés par des peignes métalliques interdigités sont réalisés par un dépôt d'aluminium d'épaisseur $1000 \AA$. Ils sont inclinés de $5^{\circ}$ pour compenser la déviation citée précédemment, et comportent 15 paires de doigts d'une périodicité de $21 \mu \mathrm{m}$ (fréquence centrale de résonance à $176 \mathrm{MHz}$ et largeur de bande à $3 \mathrm{~dB}$ de l'ordre de $10.5 \mathrm{MHz}$ ). Ces transducteurs sont enfin adaptés à $50 \Omega$, et des absorbants acoustiques sont placés de part et d'autre des zones d'interaction acousto-optique.

Les mesures des caractéristiques optiques des composants ont été réalisées d'une part à l'aide d'une diode laser DFB à $1.538 \mu \mathrm{m}$ polarisée puis focalisée à l'entrée (comme à la sortie) des guides optiques par des objectifs de microscope. Après l'interaction acousto-optique dans le composant, l'onde optique de sortie est détectée en polarisation croisée par rapport à l'entrée par un détecteur Ge. Par variation de la fréquence acoustique, et en fixant la longueur d'onde optique, nous obtenons le rendement, la largeur à mi-hauteur, et le niveau des lobes secondaires de nos différents composants. D'autre part, des caractérisations par variation de la longueur d'onde optique à fréquence acoustique fixée, ont pu être réalisées à l'aide d'un laser centre $\mathrm{F}$ accordable de 1.45 à $1.63 \mu \mathrm{m}$ au C.N.E.T. Bagneux. Ces derniers résultats sont ceux évoqués en figure $n^{\circ} 3$.

Nous présentons les résultats obtenus pour une longueur d'interaction de $27 \mathrm{~mm}\left(\mathrm{~L}=2 . \mathrm{L}_{\mathrm{c}}\right.$ dans le $3^{\text {ème }}$ cas). Nous avons exploré l'accordabilité du filtre acousto-optique sur près de $120 \mathrm{~nm}$, sans détériorer de façon importante ses caractéristiques spectrales, la largeur à mi-hauteur étant toujours inférieure à $2.2 \mathrm{~nm}$ et en conservant une puissance électrique de commande toujours inférieure à $10 \mathrm{~mW}$ (au minimum égal à $3 \mathrm{~mW}$ à $176.2 \mathrm{MHz}$ ).

${ }_{3}^{2}$ pour les trois cas de guides couplés (dans chaque cas, $\mathrm{P}_{\mathrm{a}}$ est ajusté pour obtenir $\eta=1$ au bout de $2 . \mathrm{L}_{\mathrm{C}}$ ).

3 Les diffusions ont été réalisées par le Prof. Goedgebuer et son équipe, au Laboratoire d'Optique P.M.Duffieux de I'Université de Franche-Comté (URA-CNRS 214). 

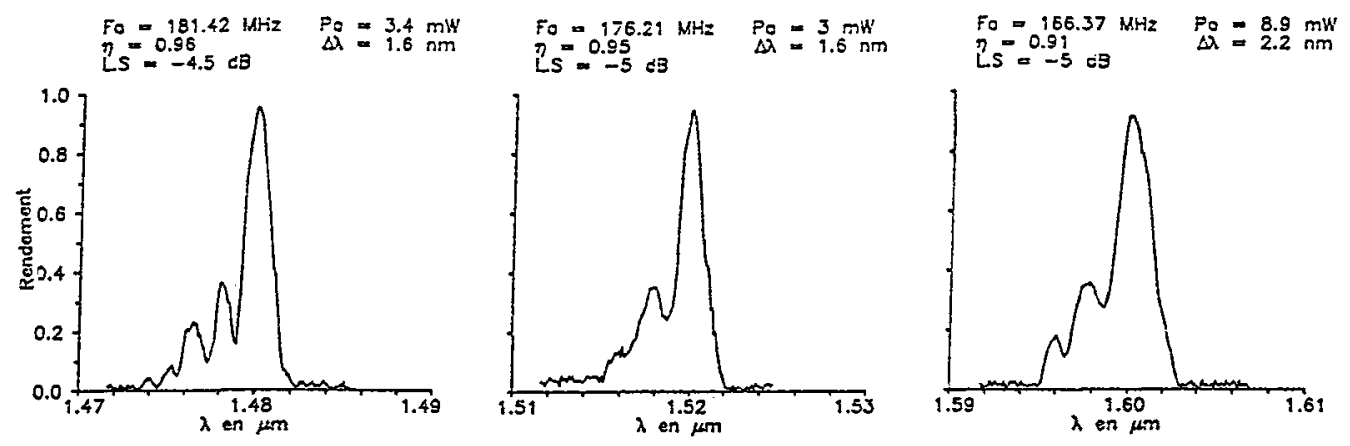

Figure $n^{\circ} 3$ : Spectres expérimentaux dans le cas d'un guide couplé ( $3^{\text {ème }}$ cas) ${ }^{4}$.

Le seul problème qui demeure reste le niveau très important des lobes secondaires, difficilement explicables compte tenu de la géométrie utilisée pour la réalisation de l'interaction. Nous pouvons écarter les explications concernant une influence parasite d'un échauffement sous les transducteurs ou les absorbants (ils ne sont pas présent au dessus du guide optique et les puissances mises en jeu restent faibles), mais pouvons envisager des déformations du guide optique lui-même lors de sa réalisation. En effet, il suffit d'une variation de $0.5 \mu \mathrm{m}$ de sa largeur, ou une variation de $35 \AA$ de son épaisseur pour induire une variation des indices optiques $\mathrm{n}_{\mathrm{e}}$ et $\mathrm{n}_{\mathrm{o}}$ de l'ordre de $1.5 \mathrm{e}^{-4}$, et expliquer le désaccord de phase à l'origine de la dissymétrie du spectre et du niveau important des lobes secondaires [7]. Des simulations numériques en cours nous permettrons sans doute de valider ces hypothèses.

D'autres composants, avec des longueurs différentes de couplage pour les guides acoustiques, ont été testés. Pour $L_{c}=6.75 \mathrm{~mm}$ et $L_{c}=9.5 \mathrm{~mm}$, nous obtenons à peu de chose près les même spectres que précédemment quelle que soit la fréquence acoustique choisie; le niveau des lobes secondaires reste sensiblement constant autour de $-5 \mathrm{~dB}$. Par contre, les largeurs à mi-hauteur ainsi que les puissances de commandes sont proportionnelles à la longueur de l'interaction. Pour le $I^{\text {er }}$ cas $\left(L=2 . L_{c}=13.5 \mathrm{~mm}\right)$, nous obtenons $\Delta \lambda=2.1 \mathrm{~nm}$ et $\mathrm{P}_{\mathrm{a}}=18.6 \mathrm{~mW}$ et pour le $2^{\mathrm{eme} e}$ cas $\left(L=2 . \mathrm{L}_{\mathrm{c}}=19 \mathrm{~mm}\right), \Delta \lambda=2 \mathrm{~nm}$ et $\mathrm{P}_{\mathrm{a}}=9 \mathrm{~mW}$ à $176 \mathrm{MHz}$.

\section{Conclusion}

Nous avons montré que l'utilisation de guides acoustiques couplés permettait d'obtenir de bons résultats quand à la largeur à mi-hauteur ou la puissance de commande du filtre. Mais des problèmes sans doute technologiques nous ont empêché de valider pour le moment lhypothèse selon laquelle ce genre de géométrie nous permettrait de diminuer le niveau des lobes secondaires. La grande accordabilité de ces composants est démontrée de façon claire. Ces filtres acousto-optiques colinéaires restent perfectibles, mais leurs potentialités devraient les rendre intéressants pour des réseaux optiques multiplexés (grande accordabilité, faible puissance de commande et faible largeur à mi-hauteur).

\section{Ce travail à bénéficié du soutien de Centre National d'Etudes des Télécommunications.} Nous tenons à remerçier Mme Sylvie Fouchet, du C.N.E.T. Bagneux, pour son aide et ses conseils au cours de ces trovaux.

[1] A.K.Roy, C.S.Tsai IEEE Photon. Tech. Lett. Vol. 4 n$^{\circ} 10$ p.1132 Oct 92

[2] S.E.Harris, R.W.Wallace Jour. Opt. Soc. America Vol.59 n ${ }^{\circ} 6$ p.744 Jun 69

[3] B.L.Heffner \& al. Elect. Lett. Vol.24 n $\mathrm{n}^{\circ} 25$ p.1562 Dec 88

[4] W.R.Trutna \& al. Opt. Lett. Vol.18 n $\mathrm{n}^{\circ} 1$ p.28 Jan 93

[5] D.A.Smith, J.J.Johnson IEEE Photon. Tech. Lett. Vol.3 n ${ }^{\circ} 10$ p.923 Oct 91

[6] D.A.Smith, J.J.Johnson Appl. Phys. Lett. Vol.61 n9 p.1025 Aug 92

[7] J.E.Baran \& al. Appl. Phys. Lett. Vol.62 n'8 p.814 Fev 93

${ }^{4} \mathrm{LC}=13.5 \mathrm{~mm}$, soit une longueur d'interaction de $L=27 \mathrm{~mm}$, à trois fréquences acoustiques différentes 166.37 , 176.21, et $181.42 \mathrm{MHz}$ avec une excursion de $\pm 10 \mathrm{~mm}$ autour de la longueur d'onde optique d'accord̉ de l'interaction ( $\Delta \lambda$ largeur à mihauteur, L.S niveau du premier lobe secondaire). 